\title{
Homomorphic image orders on combinatorial structures
}

\author{
Sophie Huczynska and Nik Ruškuc* \\ AMS classification: Primary 06A06 \\ Secondary 05C60, 06A07, 05C20, 05C05, 05A05.
}

\begin{abstract}
Combinatorial structures have been considered under various orders, including substructure order and homomorphism order. In this paper, we investigate the homomorphic image order, corresponding to the existence of a surjective homomorphism between two structures. We distinguish between strong and induced forms of the order and explore how they behave in the context of different common combinatorial structures. We focus on three aspects: antichains and partial well-order, the joint preimage property and the dual amalgamation property. The two latter properties are natural analogues of the well-known joint embedding property and amalgamation property, and are investigated here for the first time.
\end{abstract}

\section{Introduction}

Between any two relational structures $S$ and $T$, the notion of a structure-preserving map is captured by a homomorphism. Many concepts in combinatorics may fruitfully be viewed in terms of the existence (or non-existence) of certain types of homomorphisms. For example, an $r$-colouring of a graph $G$ is simply a homomorphism from $G$ into the complete graph $K_{r}$, and other graphtheoretic notions such as independence number, clique number and connectivity of a graph also possess characterizations via homomorphisms. One may also define classes of combinatorial objects by means of homomorphisms (from or into the objects). More details on this viewpoint are given in [11].

Combinatorial objects have been considered under various different orderings: two wellstudied examples are substructure order (for which we may make a further distinction between weak and induced, see [4], Section 4) and homomorphism order (see for example [6], Chapter 3). Two structures are related under the substructure order if there exists an injective homomorphism between them; a "standard" homomorphism in the case of weak substructure, and a strong homomorphism in the case of induced substructure.

By way of analogy, it is natural to consider the partial order corresponding to the existence of a surjective homomorphism between two structures; we may call this the homomorphic image order. As with the substructure order, we may distinguish between weak and induced forms. Somewhat surprisingly, this order has received very little attention in the literature. In comparison, the homomorphism order, corresponding to the existence of any homomorphism between two structures, is much-studied ([5], [10]), as are various related concepts such as the core of a graph ([3]).

\footnotetext{
${ }^{*}$ School of Mathematics and Statistics, University of St Andrews, Fife, KY16 9SS, UK, Email: Sophie.Huczynska@st-andrews.ac.uk, Nik.Ruskuc@st-andrews.ac.uk
} 
In this paper, we will investigate three forms of the homomorphic image order, focussing on three aspects: antichains and partial well order, the joint preimage property (the dual of the joint embedding property) and the dual amalgamation property (corresponding to the amalgamation property). To the best of our knowledge, the two latter properties are investigated here for the first time.

\section{Homomorphisms, orders, properties}

\subsection{Background and notation}

Let $L=\left(R_{i}\right)_{i \in I}$ be a relational signature, and let $\mathcal{C}$ be a class of structures of signature $L$. We consider structures up to isomorphism and in general we assume our structures are finite. For a structure $\mathcal{S}=\left(S, R_{i}^{\mathcal{S}}(i \in I)\right)$ and a mapping $\phi: S \rightarrow T$, we let

$$
\phi\left(R_{i}^{\mathcal{S}}\right)=\left\{\left(\phi\left(s_{1}\right), \ldots, \phi\left(s_{k}\right)\right):\left(s_{1}, \ldots, s_{k}\right) \in R_{i}^{\mathcal{S}}\right\} .
$$

We take the following definitions:

Definition 2.1. Let $\mathcal{S}=\left(S, R_{i}^{\mathcal{S}}(i \in I)\right)$ and $\mathcal{T}=\left(T, R_{i}^{\mathcal{T}}(i \in I)\right)$ be two $L$-structures, and let $\phi: S \rightarrow T$. We say that $\phi$ is:

(i) a homomorphism if $\left(s_{1}, \ldots, s_{k}\right) \in R_{i}^{\mathcal{S}} \Rightarrow\left(\phi\left(s_{1}\right), \ldots, \phi\left(s_{k}\right)\right) \in R_{i}^{\mathcal{T}}$, i.e. if $\left.\phi\left(R_{i}^{\mathcal{S}}\right) \subseteq R_{i}^{\mathcal{T}}\right|_{\phi(S)}$;

(ii) a strong homomorphism if $\phi$ is a homomorphism and $\phi\left(R_{i}^{\mathcal{S}}\right)=\left.R_{i}^{\mathcal{T}}\right|_{\phi(S)}$;

(iii) an M-strong homomorphism if $\phi$ is a homomorphism such that $\left(s_{1}, \ldots, s_{k}\right) \in R_{i}^{\mathcal{S}} \Leftrightarrow\left(\phi\left(s_{1}\right), \ldots, \phi\left(s_{k}\right)\right) \in$ $R_{i}^{\mathcal{T}}$, i.e. $\phi\left(R_{i}^{\mathcal{S}}\right)=\left.R_{i}^{\mathcal{T}}\right|_{\phi(S)}$ and $\phi\left(\overline{R_{i}^{\mathcal{S}}}\right)=\left.\overline{R_{i}^{\mathcal{T}}}\right|_{\phi(S)}$.

The " $\mathrm{M}$ " in the final definition refers to the model theoretic definition of a strong embedding (see, for example, [8], Chapter 1). Our definition of strong homomorphism requires that every related $k$-tuple in $\phi(S)$ must be the image of at least one related $k$-tuple in $S$ but, in contrast to the model theoretic definition, it does not require that every preimage of a related $k$-tuple in $\phi(S)$ must be a related $k$-tuple in $S$.

We make the following further definitions:

Definition 2.2. A homomorphism is an embedding if it is injective, and is an epimorphism if it is onto. Like any other homomorphisms, embeddings and epimorphisms may be strong or Mstrong. An isomorphism is an M-strong bijection.

It is clear that every M-strong homomorphism is strong. Moreover, an embedding $\phi: S \rightarrow T$ is M-strong if and only if it is strong. Considering the three strengths of homomorphisms for injections and surjections leads to six partial orders on finite structures, of which only five are distinct by the previous observation. In addition to the customary notation $\preceq$ appropriately subscripted, we will also find it convenient to have a letter notation for parts of the text where we treat the order as a set.

Definition 2.3. (i) Substructure order $\preceq_{\mathrm{S}}$ or $O_{\mathrm{S}}: A \preceq_{\mathrm{S}} B$ if there exists an embedding $\phi: A \rightarrow$ $B$;

(ii) induced substructure order $\preceq_{\text {IS }}$ or $O_{\text {IS }}: A \preceq_{\text {IS }} B$ if there exists a strong embedding $\phi: A \rightarrow$ $B$; 
(iii) homomorphic image order $\preceq_{\mathrm{H}}$ or $O_{\mathrm{H}}: A \preceq_{\mathrm{H}} B$ if there exists an epimorphism $B \rightarrow A$;

(iv) induced homomorphic image order $\preceq_{\mathrm{IH}}$ or $O_{\mathrm{IH}}: A \preceq_{\mathrm{IH}} B$ if there exists a strong epimorphism $B \rightarrow A$;

(v) M-induced homomorphic image order $\preceq_{\mathrm{MH}}$ or $O_{\mathrm{MH}}: A \preceq_{\mathrm{MH}} B$ if there exists an M-strong epimorphism $B \rightarrow A$.

Note that in all these definitions the direction of homomorphisms is chosen so as to ensure that $A \preceq B$ implies $|A| \leq|B|$.

Lemma 2.4. Let $\mathcal{C}$ be a collection of finite relational structures of the same signature.

(i) All of $\mathrm{O}_{\mathrm{S}}, \mathrm{O}_{\mathrm{IS}}, \mathrm{O}_{\mathrm{H}}, \mathrm{O}_{\mathrm{IH}}$ and $\mathrm{O}_{\mathrm{MH}}$ are partial orders on $\mathcal{C}$;

(ii) $\mathrm{O}_{\mathrm{IS}} \subseteq \mathrm{O}_{\mathrm{S}}$ and $\mathrm{O}_{\mathrm{MH}} \subseteq \mathrm{O}_{\mathrm{IH}} \subseteq \mathrm{O}_{\mathrm{H}}$.

The inclusions (ii) follow immediately from Definition 2.3. Reflexivity and transitivity for each of the relations is straightforward, while antisymmetry relies on the finiteness assumption. Without this assumption, the above relations are only quasiorders. Note that $O_{\mathrm{S}}$ and $O_{\mathrm{IS}}$ are the usual substructure and induced substructure orderings; the remaining three will be the focus of the present article.

\subsection{Types of structures}

Our aim is to investigate how the above orders behave in the context of different common combinatorial structures; in this subsection, we introduce the structures which will be considered.

A digraph is simply a set $D$ with a binary relation $E(D)$. A related pair $(x, y) \in E(D)$ is called a (directed) edge. A digraph homomorphism $\phi: D_{1} \rightarrow D_{2}$ maps edges to edges; $\phi$ is strong if it maps $E\left(D_{1}\right)$ onto $E\left(D_{2}\right)$ and is M-strong if it also maps non-edges to non-edges.

In combinatorics there are numerous graph-like specializations of digraphs. In this paper we will consider the following.

A graph is a digraph $G$ in which the edge relation $E(G)$ is symmetric. Furthermore, we will insist that $E(G)$ is either irreflexive or reflexive. This choice affects the notion of homomorphisms: in the irreflexive representation a homomorphism may not "collapse" an edge to a single point, while in the reflexive representation both edges and non-edges may be collapsed.

A tree is a connected graph with no cycles. Again, we will consider trees in two different representations, reflexive and irreflexive. At times, we will also consider rooted trees, where homomorphisms are required to map root to root.

A tournament is a digraph $T$ in which for any two distinct $x, y \in T$, precisely one of $(x, y)$ or $(y, x)$ is an edge. Again, we consider reflexive and irreflexive tournaments. In the irreflexive case, since a homomorphism may not collapse an edge, every homomorphism is injective.

An equivalence relation is a digraph $X$ with a reflexive, symmetric and transitive edge set $E(X)$. A mapping $\phi: X_{1} \rightarrow X_{2}$ is a homomorphism precisely if it maps the equivalence classes of $E\left(X_{1}\right)$ into equivalence classes of $E\left(X_{2}\right)$. A homomorphism $\phi$ is strong if every pair of elements in $\operatorname{im} \phi$ which lie in the same equivalence class of $E\left(X_{2}\right)$ possesses a pair of preimages which lie in the same equivalence class of $E\left(X_{1}\right)$. Finally, a homomorphism is M-strong if at most one equivalence class of $E\left(X_{1}\right)$ is mapped into each equivalence class of $E\left(X_{2}\right)$.

A poset is a digraph $P$ in which the relation $E(P)$ (usually written $\leq$ ) is anti-symmetric and transitive. We consider reflexive and irreflexive posets. Note that an M-strong homomorphism in the reflexive case is necessarily injective because of anti-symmetry. 
A linear order is a partial order $L$ in which for any two distinct $x, y \in L$ we have $x \leq y$ or $y \leq x$. A mapping $\phi: L_{1} \rightarrow L_{2}$ between two linear orders is a homomorphism if and only if all preimages $\phi^{-1}(y)\left(y \in L_{2}\right)$ are contiguous subsets of $L_{1}$ and if $y_{1}<y_{2}\left(y_{1}, y_{2} \in L_{2}\right)$ and $x_{i} \in \phi^{-1}\left(y_{i}\right)$ imply $x_{1}<x_{2}$. It is straightforward to see that every homomorphism is strong, but that only the injective ones are M-strong.

A word over alphabet $A$ is a relational structure $\left(W, \leq, f_{a}(a \in A)\right)$ where $\leq$ is a linear order and $f_{a}(a \in A)$ are unary relations such that for each $w \in W, w \in f_{a}$ is true for precisely one $a \in A$. Given such a structure, if $W=\left\{w_{1}, \ldots, w_{k}\right\}$ with $w_{1}<\cdots<w_{k}$ we write $W=a_{1} \ldots a_{k}$ where $w_{i} \in f_{a_{i}}$, a sequence of letters from the alphabet. A mapping $\phi: W_{1} \rightarrow W_{2}$ between two such relational structures is a homomorphism if the preimages $\phi^{-1}(y)$ of points in $W_{2}$ are appropriately ordered contiguous segments of $W$, as in the linear orders case and, in addition, all $x \in \phi^{-1}(y)$ belong to the same $f_{a}$. Thus, if $W_{1}=a_{1} \ldots a_{k}$ and $W_{2}=a_{1}^{\prime} \ldots a_{l}^{\prime}$ are written as sequences, $\phi$ can be thought of as a "process" which identifies contiguous occurrences of the same letter in $W_{1}$ and yields a subsequence (subword) of $W_{2}$. As with linear orders, every homomorphism is strong but only the injective ones are M-strong.

A permutation is a relational structure $\left(P, \leq_{1}, \leq_{2}\right)$ where $\leq_{1}, \leq_{2}$ are linear orders. A mapping $f: P_{1} \rightarrow P_{2}$ between two permutations is a homomorphism if the preimages of points in $P_{2}$ are contiguous under both $\leq_{1}$ and $\leq_{2}$. Such sets are known as intervals, see [1]. Yet again, all homomorphisms are strong and only the injective ones are M-strong. All these assertions follow from their counterparts above.

\subsection{Relationships between orders}

In general, the three orders are distinct, with $\mathrm{O}_{\mathrm{MH}} \subseteq \mathrm{O}_{\mathrm{IH}} \subseteq \mathrm{O}_{\mathrm{H}}$. For certain classes of combinatorial structures, some of the orders may coincide, or reduce to isomorphism. Table 1 summarizes this by indicating when all homomorphisms are strong or M-strong (entries "hom.") and when some types of homomorphism are necessarily injective (entries "inj.").

Table 1: Summary of certain order properties

\begin{tabular}{|l|ccc|ccc|}
\hline \hline Class $\mathcal{C}$ & hom. & strong hom. & M-strong hom. & $O_{\mathrm{H}}$ & $\mathrm{O}_{\mathrm{IH}}$ & $O_{\mathrm{MH}}$ \\
\hline Tournaments & & & & & & \\
(reflexive) & & hom. & inj. & & $O_{\mathrm{H}}$ & $\cong$ \\
Tournaments & & & & & \\
(irreflexive) & inj. & inj. & inj. & $\cong$ & $\cong$ & $\cong$ \\
Trees (reflexive) & & hom. & Lemma 2.6 & & $O_{\mathrm{H}}$ & \\
Trees (irreflexive) & & hom. & inj. & & $O_{\mathrm{H}}$ & $\cong$ \\
Posets (reflexive) & & & inj. & & \\
Linear orders & & hom. & inj. & & $O_{\mathrm{H}}$ & $\cong$ \\
Permutations & & hom. & inj. & & $O_{\mathrm{H}}$ & $\cong$ \\
Words & & hom. & inj. & & $O_{\mathrm{H}}$ & $\cong$ \\
\hline
\end{tabular}

We justify the claim in the table concerning trees.

Lemma 2.5. For the class of trees (reflexive or irreflexive), every homomorphism is strong and hence $\mathrm{O}_{\mathrm{H}}=\mathrm{O}_{\mathrm{IH}}$.

Proof. Let $\phi: S \rightarrow T$ be a homomorphism of trees. Let $(u, v)$ be an edge in $\phi(S)(u \neq v)$. Let $w_{u}, w_{v}$ be preimages of $u, v$ in $S$. Let $w_{1}, \ldots, w_{k}$ be a path in $S$ connecting $w_{u}$ and $w_{v}$. By 
considering the image of this path under $\phi$, take the shortest subpath connecting $u$ and $v$; let this be $\phi\left(w_{i}\right), \phi\left(w_{i+1}\right), \ldots, \phi\left(w_{j}\right)$ where $\phi\left(w_{i}\right)=u$ and $\phi\left(w_{j}\right)=v$. Note that $\phi\left(w_{s}\right) \neq u, v$ for $i<s<j$. We cannot have $j>i+1$, since then $\phi\left(w_{i}\right), \ldots, \phi\left(w_{j}\right), u$ would be a cycle. Hence $j=i+1$ and $\left(w_{i}, w_{i+1}\right)$ is an edge in $S$ which is mapped onto $(u, v)$ in $T$.

Furthermore, we observe that M-strong epimorphisms of reflexive trees are "almost" isomorphisms.

Lemma 2.6. A mapping $\phi: T \rightarrow U$ is an M-strong epimorphism of reflexive trees if and only if $\phi$ is an isomorphism or $U$ is trivial and $|T|=2$.

Proof. Suppose $\phi$ is not an isomorphism and let $x, y \in T$ be such that $\phi(x)=\phi(y)=u \in U$. Suppose $|U|>1$, and let $v \in U$ be any vertex adjacent to $u$. Let $z \in T$ be any preimage of $v$. Then $\{x, y, z\}$ form a triangle, a contradiction. On the other hand, if $U$ is trivial, then $T$ is complete, and hence a tree only if $|T| \leq 2$.

\subsection{PWO and join properties}

As described in the Introduction, for each of the three homomorphic image orders, our investigations will focus on three aspects, reflecting some fundamental structural properties of posets.

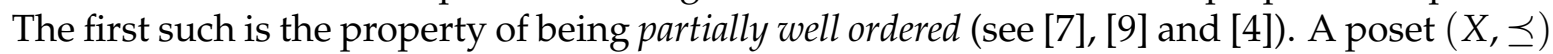
has this property if it has no infinite antichains and no infinite descending chains. In our context there can never be infinite descending chains, because of size considerations, and we are left to investigate the existence or otherwise of infinite antichains.

A poset $(X, \preceq)$ is said to have the join property if for any two $x, y \in X$ there exists $z \in X$ such that $x \preceq z$ and $y \preceq z$. When applied to the order $\preceq_{\text {IS }}$ this notion becomes the well known joint embedding property (JEP): a class $\mathcal{C}$ has JEP if for any two $A, B \in \mathcal{C}$ there exists $C \in \mathcal{C}$ into which both $A$ and $B$ strongly embed. Paralleling this, each of our homomorphic image orders gives rise to a join-type property. We say that $\mathcal{C}$ has the joint preimage property (JPP) if for any $A, B \in \mathcal{C}$ there exists $C \in \mathcal{C}$ which has both $A$ and $B$ as homomorphic images. By strengthening homomorphisms to strong- or M-strong homomorphisms we obtain the strong- and M-strong JPP.

A property related to the JEP is the amalgamation property (AP), which stipulates that for any $A, B, C \in \mathcal{C}$, with strong embeddings $f: A \rightarrow B$ and $g: A \rightarrow C$, there exist $D \in \mathcal{C}$ and strong embeddings $h: B \rightarrow D$ and $k: C \rightarrow D$ such that $h f=k g$. We will term the analogue in our context the dual amalgamation property (DAP); it requires that for any $A, B, C \in \mathcal{C}$, with epimorphisms $f: B \rightarrow A$ and $g: C \rightarrow A$, there exist $D \in \mathcal{C}$ and epimorphisms $h: D \rightarrow B$ and $k: D \rightarrow C$ such that $f h=g k$. Again, we also have the strong and M-strong variants of DAP.

The customary diagrams illustrating the definitions of JEP, JPP, AP and DAP are shown in Figure 1

We present our material by proceeding property by property; however, to facilitate comparison of the main results on an order-by-order basis, three tables (one corresponding to each order) are provided at the end of the paper.

\section{Antichains and partial well order}

This section addresses the question: for a given class $\mathcal{C}$ under a given homomorphic image order, can we construct an infinite antichain in $\mathcal{C}$, or is $\mathcal{C}$ PWO? For most choices of class and order, we find that an appropriate infinite antichain can be constructed, notable exceptions being trees and 

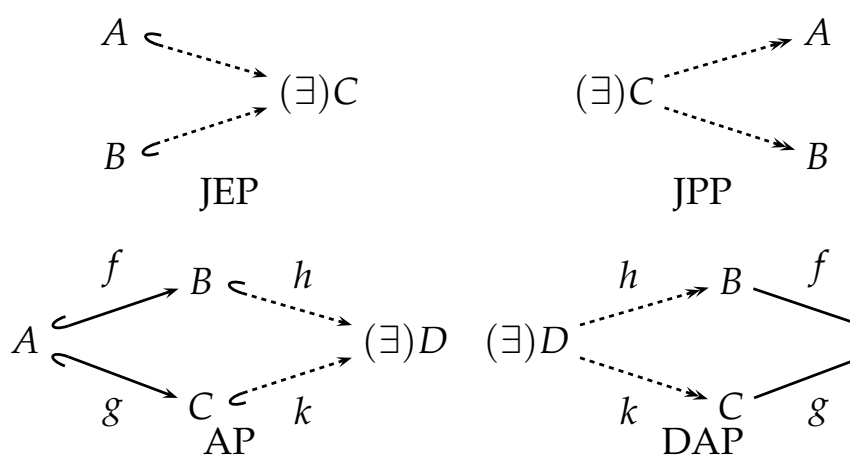

Figure 1: JEP, JPP, AP and DAP.

equivalence relations in the $O_{\mathrm{IH}}$ and $\mathrm{O}_{\mathrm{H}}$ cases. For a negative answer to the PWO question, it of course suffices to exhibit the antichain corresponding to the weakest such order, though we will also discuss antichains which differentiate between the three orders.

\subsection{Digraphs}

We begin by considering digraphs.

Theorem 3.1. Let $\mathcal{C}$ be the class of digraphs.

(i) Let $P=\left\{P_{n}: n(\geq 4) \in \mathbb{N}\right\}$, where digraph $P_{n}$ has vertices $\{1, \ldots, n\}$ and edges

$$
\{(i, i+1): 1 \leq i \leq n-1\} \cup\{(i, i): 1 \leq i \leq n\}
$$

(a directed path). Then $P$ is an antichain with respect to $O_{\mathrm{MH}}$ but not with respect to $\mathrm{O}_{\mathrm{IH}}$.

(ii) Let $G=\left\{G_{n}: n(\geq 4) \in \mathbb{N}\right\}$, where $G_{n}$ has vertices $\{1, \ldots, n\}$ and edges

$$
\{(i, i+1): 1 \leq i \leq n-1\} \cup\{(n-2, n),(n, n)\}
$$

(see Figure 2). Then $G$ is an antichain with respect to $O_{\mathrm{IH}}$ but not with respect to $\mathrm{O}_{\mathrm{H}}$.

(iii) Let $K=\left\{K_{n}: n(\geq 2) \in \mathbb{N}\right\}$, where $K_{n}$ has vertices $\{1, \ldots, n\}$ and edges $\{(i, j): 1 \leq i<j \leq n\}$ (a linear tournament). Then $\mathrm{K}$ is an antichain w.r.t. $\mathrm{O}_{\mathrm{H}}$.

Proof. (i) For every pair of distinct vertices $\{x, y\}$ in $P_{n}$, there is some vertex $z$ connected to precisely one of $\{x, y\}$, and so $x$ and $y$ cannot be mapped by an M-strong homomorphism to the same image point. Hence $P_{n}$ is a minimal element under $O_{\mathrm{MH}}$. Consider the mapping from $P_{n}$ to $P_{n-1}$ given by $i \mapsto i$ for $1 \leq i \leq n-1$ and $n \mapsto n-1$ : it is a strong epimorphism. To see that it is not M-strong, observe that non-edge $(n-2, n)$ and edge $(n-2, n-1)$ are both mapped to edge $(n-2, n-1)$.

(ii) We first establish that the mapping $\phi: G_{n} \rightarrow G_{m}(n>m)$ given by

$$
\phi(x)= \begin{cases}x, & x \leq m \\ m, & x>m\end{cases}
$$

is a homomorphism, but not a strong homomorphism. We check that $\phi$ maps edges to edges: for $1 \leq i \leq n-1$, edge $(i, i+1)$ is mapped to $(i, i+1)$ if $i<m$ and to $(m, m)$ if $i \geq m$. Edge 


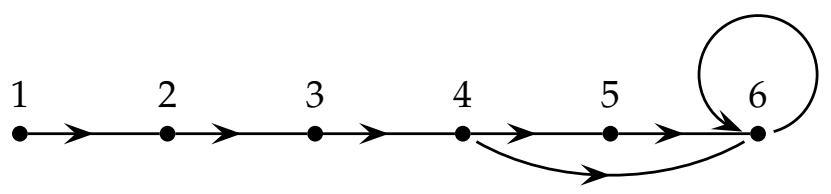

Figure 2: Digraph $G_{6}$ from the proof of Theorem 3.1

$(n, n)$ is mapped to $(m, m)$ and edge $(n-2, n)$ is mapped to $(m-1, m)$ if $n=m+1$ and $(m, m)$ otherwise. It is clear that $\phi$ is onto; however, since edge $(m-2, m)$ in $G_{m}$ is not the image of an edge in $G_{n}, \phi$ is not strong.

We now show that $\phi$ is the unique onto homomorphism from $G_{n}$ to $G_{m}$. Let $\theta: G_{n} \rightarrow G_{m}$ $(n>m)$ be an onto homomorphism. We claim that $\theta(1)=1$. Suppose $\theta(1)=k \neq 1$. Let $j \in G_{n}$ be arbitrary. Since there is a directed path in $G_{n}$ from 1 to $j$, in $G_{m}$ we must have a directed path from $\theta(1)=k$ to $\theta(j)$. Hence $\theta(j) \neq 1$, and so $\theta$ is not onto. For $i=1, \ldots, m-3$ there is a unique edge, namely $(i, i+1)$, coming out of $i$ in both $G_{m}$ and $G_{n}$. Hence $\theta(i)=i$ for $i=1,2, \ldots, m-2$. In $G_{n}$, there is only one edge coming out of $m-2$, namely $(m-2, m-1)$. In $G_{m}$, there are two: $(m-2, m-1)$ and $(m-2, m)$. Hence $\theta(m-1) \in\{m-1, m\}$. If $\theta(m-1)=m$ then $\theta(m)=\theta(m+1)=\cdots=\theta(n)=m$ because of edges $(m-1, m),(m, m+1), \ldots,(n-1, n)$. But then $\theta(x) \neq m-1$, a contradiction. Hence $\theta(m-1)=m-1$ and then $\theta(m)=m$. Thus $\theta$ is the mapping $\phi$ defined above. Since $\phi$ is not strong, it follows that $G$ is an antichain under $O_{\mathrm{IH}}$.

(iii) Suppose $\phi: K_{m} \rightarrow K_{n}(m>n)$ is any mapping. Then there are $i, j \in[m](i<j)$ such that $\phi(i)=\phi(j)=k$. But then the edge $(i, j)$ in $K_{m}$ is mapped to a non-edge $(k, k)$ in $K_{n}$. Hence $\phi$ is not a homomorphism, proving that $\left\{K_{n}: n \in \mathbb{N}\right\}$ is an antichain.

\subsection{Graphs}

Note that, in the irreflexive case, every complete graph is minimal.

Theorem 3.2. The class of irreflexive graphs has infinitely many minimal elements with respect to $\mathrm{O}_{\mathrm{H}}$ and hence is not PWO.

In the reflexive case, by contrast, the trivial graph is the unique minimal element. Nonetheless, we have:

Theorem 3.3. The class of reflexive graphs is not $P W O$ with respect to $O_{\mathrm{H}}$.

Proof. Let $G_{n}$ be the graph on $2 n$ vertices $\{1, \ldots, 2 n\}$ such that $(2 i-1,2 i)$ is a non-edge ( $i=$ $1, \ldots, n)$ and all other pairs of vertices are edges; see Figure 3. Let $\phi: G_{k} \rightarrow G_{l}(k>l)$ be an onto mapping. For some $r$ in $G_{l}, r=\phi(p)=\phi(q)$ for distinct $p, q$ in $G_{k}$ (without loss, we assume that $r=1)$. Recall that $(1,2)$ is a non-edge in $G_{l}$ and consider a preimage $s$ of 2 . At least one of $(p, s)$, $(q, s)$ must be an edge, hence $\phi$ is not a homomorphism.

Remark 3.4. Some common families of graphs are antichains with respect to $\preceq_{\mathrm{MH}}$, but not with respect to the two weaker orders. For example, the set of paths on 4 or more vertices is an antichain with respect to $O_{\mathrm{MH}}$ but not with respect to $O_{\mathrm{IH}}$ (in both the reflexive and irreflexive 


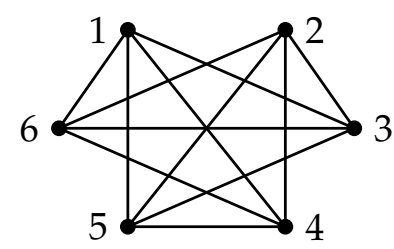

Figure 3: Graph $G_{3}$ from the proof of Theorem 3.3
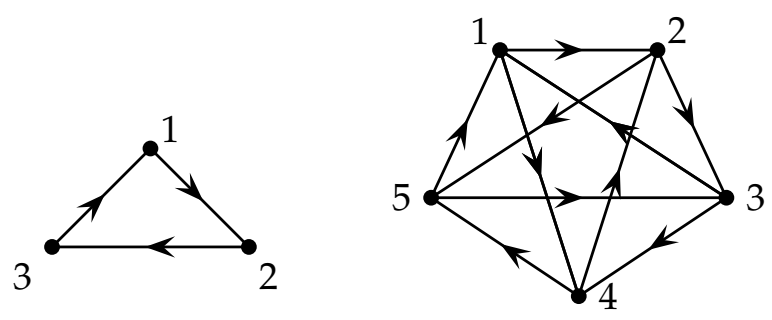

Figure 4: $T_{3}$ and $T_{5}$ of Proposition 3.5

setting). Infinite families of graphs which are antichains with respect to $O_{\mathrm{IH}}$ but not with respect to $O_{\mathrm{H}}$ do not arise so naturally.

\subsection{Tournaments}

Recall that, for irreflexive tournaments, all homomorphism are injective (see Table 1).

Proposition 3.5. Let $n \in \mathbb{N}$ be odd. The tournament $T_{n}$ on $n$ vertices $\{1, \ldots, n\}$ given by the rule: for $1 \leq i<j \leq n$,

$$
\begin{array}{lll}
i \rightarrow j & \text { if } i \neq j \quad & (\bmod 2) \\
j \rightarrow i & \text { if } i \equiv j & (\bmod 2)
\end{array}
$$

(see Figure 4) has no proper non-trivial homomorphic image within the class of reflexive or irreflexive tournaments.

Proof. It is immediate that a directed triangle $\left(T_{3}\right)$ has no non-trivial homomorphic image. We show that every edge of $T_{n}$ is in some directed triangle. To see this, consider $i, j \in[n]$ with $i<j$. If $i$ and $j$ are of the same parity, then $j \rightarrow i$. Take any $k$ of opposite parity from both, such that $i<k<j$; then $i \rightarrow k$ and $k \rightarrow j$. If $i$ and $j$ are of opposite parity, then $i \rightarrow j$. If $j<n$ then $\{i, j, j+1\}$ form a triangle, while if $j=n$ then $\{1, i, j\}$ form a triangle. Since it is not possible to collapse a directed triangle, the result follows.

Theorem 3.6. The class of reflexive (or irreflexive) tournaments is not $P W O$ with respect to $O_{\mathrm{H}}$.

Proof. The reflexive case is a consequence of Proposition 3.5, while the irreflexive case follows from the non-existence of proper epimorphisms.

\subsection{Posets}

Similarly to the case of graphs, in the class of irreflexive posets, every chain is a minimal element. 


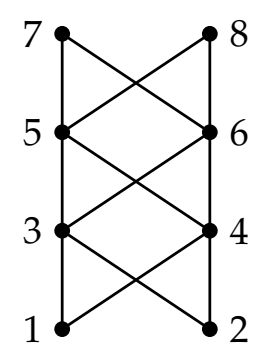

Figure 5: Poset $B_{4}$ from the proof of Theorem 3.8

Theorem 3.7. The class of irreflexive posets has infinitely many minimal elements with respect to $O_{\mathrm{H}}$ and hence is not PWO.

For the reflexive case we have:

Theorem 3.8. The class of reflexive posets is not PWO with respect to $O_{\mathrm{H}}$.

Proof. Let $B_{n}$ be the poset on $2 n$ vertices $\{1, \ldots, 2 n\}$ such that, for each $i=2, \ldots, n-1$ : vertices $2 i-1$ and $2 i$ are incomparable; each covers $2 i-2$ and $2 i-3$; and each is covered by $2 i+1$ and $2 i+2$; see Figure 5. Let $\phi: B_{k} \rightarrow B_{l}(k>l)$ be an onto mapping. For some $r$ in $B_{l}, r=\phi(p)=\phi(q)$ for distinct $p, q$ in $B_{k}$. There is a unique element $s$ in $B_{l}$, incomparable to $r$. Let $u$ be a preimage of $s$. At least one of $p, q$ is comparable to $u$, say this is $p$. Then $\phi$ maps the comparable pair $(p, u)$ to an incomparable pair $(r, s)$, and so $\phi$ is not a homomorphism.

As with graphs, it is easy to find families of posets which form antichains with respect to the strongest order but not with respect to the two weaker orders. For example, let $\mathcal{F}=\left\{F_{1}, F_{2}, \ldots\right\}$ where $F_{n}$ is the poset on $2 n$ vertices $\{1, \ldots, 2 n\}$ such that $2 i-1$ covers $2 i(i=1, \ldots, n)$ and all other pairs of elements are incomparable. Then (in both the reflexive and irreflexive setting) $\mathcal{F}$ is an antichain with respect to $O_{\mathrm{MH}}$ but not with respect to $O_{\mathrm{IH}}$. Again, examples distinguishing between $O_{\mathrm{IH}}$ and $O_{\mathrm{H}}$ do not arise so naturally.

\subsection{Trees}

We now consider a class of structure which provides a positive answer to our PWO question, namely trees. We will prove our result for irreflexive trees; the case of reflexive trees then follows. Our strategy involves proceeding via rooted trees. Recall that a rooted tree $T$ may conveniently be decomposed into levels; $L_{0}$ is the root, and $L_{i+1}$ is the set of vertices from $T \backslash\left\{L_{0} \cup \ldots \cup L_{i}\right\}$ adjacent to elements in $L_{i}$. The depth is the largest $d$ such that $L_{d} \neq \varnothing$.

Let $\mathcal{T}$ be the class of irreflexive trees, and let $\mathcal{T}^{\bullet}$ be the class of irreflexive rooted trees.

Lemma 3.9. Let $T \in \mathcal{T} \bullet$ be a rooted tree of depth d with root $r$, and let $P$ be a path with vertices $p_{0}, \ldots, p_{e}$ and edges $\left(p_{i}, p_{i+1}\right), i=0, \ldots, e-1$. If $d \geq e$ then there is an epimorphism $\phi: T \rightarrow P$ with $\phi(r)=p_{0}$.

Proof. Let $L_{0}=\{r\}, L_{1}, \ldots, L_{d}$ be the levels of $T$. The mapping $\phi: T \rightarrow P$ defined by

$$
\phi(v)=\left\{\begin{array}{lll}
p_{i}, & \text { if } v \in L_{i}, & 0 \leq i \leq e \\
p_{e}, & \text { if } v \in L_{i}, & e<i \leq d \text { and } i \equiv e \quad(\bmod 2) \\
p_{e-1}, & \text { if } v \in L_{i}, & e<i \leq d \text { and } i \equiv e+1 \quad(\bmod 2)
\end{array}\right.
$$

has the desired properties. 


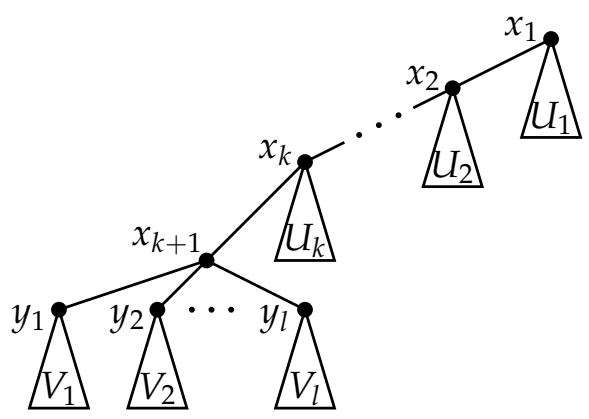

Figure 6: The construction of $\tau(P)$. Here $P=\left(U_{1}, \ldots, U_{k} ; V_{1}, \ldots, V_{l}\right)$, where $U_{1}, \ldots, U_{k}, V_{1}, \ldots, V_{l}$ are trees rooted at $x_{1}, \ldots, x_{k}, y_{1}, \ldots, y_{l}$ respectively.

Lemma 3.10. Let $\mathcal{C}^{\prime}$ be the class of irreflexive rooted trees. For every non-trivial $S \in \mathcal{C}^{\prime}$, the set $\operatorname{Av}(S)=$ $\left\{T \in \mathcal{C}^{\prime}: S \swarrow_{\mathrm{H}} T\right\}$ is PWO.

Proof. Induction on $|S|$, the case $|S|=2$ being trivial by Lemma 3.9. Suppose $|S|>2$. Let $v_{0}$ be the root of $S$, let $d=\operatorname{depth}(S)$ and let $v_{0}, v_{1}, \ldots, v_{d}$ be any path in $S$ of length $d$ (note $v_{d}$ must be a leaf). Let $S^{\prime}$ be the tree $S$ with $v_{d}$ removed. By induction, $\operatorname{Av}\left(S^{\prime}\right)$ is $\mathrm{PWO}$.

Consider an arbitrary $T \in \operatorname{Av}(S)$, with root $r$. Let $c_{1}, \ldots, c_{t}$ be the children of $r$. Let $T_{i}$ be the subtree of $T$ rooted in $c_{i}(i=1, \ldots, t)$; call these the principal subtrees. We claim that one of the following holds:

(i) all $T_{i}$ are in $\operatorname{Av}\left(S^{\prime}\right)$; or

(ii) if, for some $i, S^{\prime} \preceq_{\mathrm{H}} T_{i}$, then removing $T_{i}$ from $T$ yields a tree in $\operatorname{Av}\left(S^{\prime}\right)$.

For, suppose not, so that (say) each of $T_{1}$ and $T \backslash T_{1}$ has $S^{\prime}$ as a homomorphic image. Let $\phi: T \backslash T_{1} \rightarrow S^{\prime}$ be an epimorphism. Note that $\operatorname{depth}\left(T_{1}\right) \geq \operatorname{depth}\left(S^{\prime}\right) \geq d-1$ because we are dealing with homomorphisms of rooted trees. By Lemma 3.9, there is a homomorphism $\psi: T_{1} \rightarrow S$ with $\psi\left(T_{1}\right)=\left\{v_{1}, \ldots, v_{d}\right\}$ and $\psi\left(c_{1}\right)=v_{1}$. Define $\pi: T \rightarrow S$ as follows:

$$
\pi(v)= \begin{cases}\psi(v), & v \in T_{1} \\ \phi(v), & v \notin T_{1} .\end{cases}
$$

This is a homomorphism because $\phi$ and $\psi$ are, and $\pi(r)=\phi(r)=v_{0}, \pi\left(c_{1}\right)=\psi\left(c_{1}\right)=v_{1}$ and $v_{0}, v_{1}$ are adjacent in $S$. It is also onto because $\pi(T)=\phi\left(T \backslash T_{1}\right) \cup \psi\left(T_{1}\right)=S^{\prime} \cup\left\{v_{1}, \ldots, v_{d}\right\}=S$. This contradicts $T \in \operatorname{Av}(S)$.

By a repeated application of this claim, we see that to every $T \in \operatorname{Av}(S)$, we can associate a pair of sequences $\left(U_{1}, \ldots, U_{k} ; V_{1}, \ldots, V_{l}\right)$ of members of $\operatorname{Av}\left(S^{\prime}\right)$. Starting with $T$, and supposing (ii) holds, we let $U_{1}=T \backslash T_{i} \in \operatorname{Av}\left(S^{\prime}\right)$ and $T^{(1)}=T_{i}$. We repeat this process, constructing $U_{1}, \ldots, U_{k} \in \operatorname{Av}\left(S^{\prime}\right)$ and $T^{(1)}, \ldots, T^{(k)}$ having $S^{\prime}$ as a homomorphic image, until all principal subtrees $V_{1}, \ldots, V_{l}$ of $T^{(k)}$ lie in $\operatorname{Av}\left(S^{\prime}\right)$, at which stage we append them to $U_{1}, \ldots, U_{k}$ and stop the process. Conversely, every pair of sequences $P=\left(U_{1}, \ldots, U_{k} ; V_{1}, \ldots, V_{l}\right)$ defines a unique tree $\tau(P)$, obtained by connecting the trees $U_{1}, \ldots, U_{k}, V_{1}, \ldots, V_{l}$ as indicated in Figure 6.

Denote by $\mathcal{P}$ the set of all such pairs of sequences. We define the following ordering on $\mathcal{P}$ :

$$
\left(U_{1}, \ldots, U_{k} ; V_{1}, \ldots, V_{l}\right) \leq\left(U_{1}^{\prime}, \ldots, U_{m}^{\prime} ; V_{1}^{\prime}, \ldots, V_{n}^{\prime}\right)
$$

if and only if 


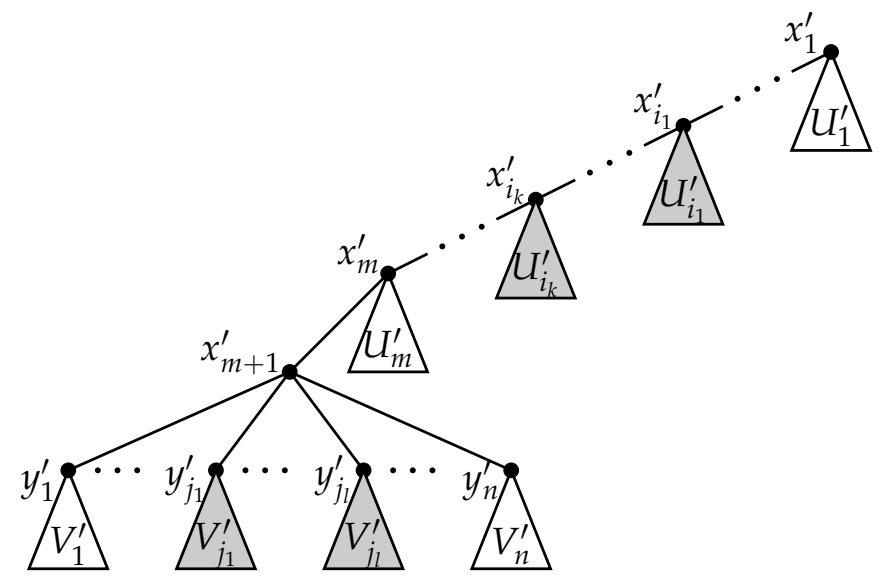

Figure 7: $\tau\left(P^{\prime}\right)$, where $P^{\prime}=\left(U_{1}^{\prime}, \ldots, U_{m}^{\prime} ; V_{1}^{\prime}, \ldots, V_{n}^{\prime}\right)$. The shaded subtrees $U_{i_{1}}^{\prime}, \ldots, U_{i_{k}}^{\prime}, V_{j_{1}}^{\prime}, \ldots, V_{j_{l}}^{\prime}$ are mapped onto $U_{1}, \ldots, U_{k}, V_{1}, \ldots, V_{l}$ respectively via $\zeta$.

- $k \equiv m(\bmod 2)$

and there exist $1 \leq i_{1}<\ldots<i_{k} \leq m$ and $1 \leq j_{1}<\ldots<j_{l} \leq n$ such that

- $U_{s} \preceq_{\mathrm{H}} U_{i_{s}}^{\prime}, \quad s=1, \ldots, k$;

- $V_{s} \preceq_{\mathrm{H}} V_{j_{s}}^{\prime}, \quad s=1, \ldots, l$;

- $i_{s} \equiv s(\bmod 2)$.

Since this ordering is derived from the usual subsequence ordering by imposing a couple of finitary restrictions, a standard multiple application of Higman's Theorem ([7]) yields that $\mathcal{P}$ is PWO.

Next, we prove that $\tau$ preserves ordering. Let

$$
P=\left(U_{1}, \ldots, U_{k} ; V_{1}, \ldots, V_{l}\right) \leq P^{\prime}=\left(U_{1}^{\prime}, \ldots, U_{m}^{\prime} ; V_{1}^{\prime}, \ldots, V_{n}^{\prime}\right)
$$

with $\tau(P)$ and $\tau\left(P^{\prime}\right)$ as shown in Figures 6 and 7. By definition, there exist epimorphisms $\mu_{s}$ : $U_{i_{s}}^{\prime} \rightarrow U_{s}(s=1, \ldots, k)$ and $v_{s}: V_{j_{s}}^{\prime} \rightarrow V_{s}(s=1, \ldots, l)$. We shall exhibit an epimorphism $\zeta$ sending $\tau\left(P^{\prime}\right)$ to $\tau(P)$. The essential idea of $\zeta$ is that it maps each $U_{i_{s}}^{\prime}$ onto $U_{s}$ and each $V_{j_{s}}^{\prime}$ onto $V_{s}$, while every other $U_{i}^{\prime}$ and $V_{i}^{\prime}$ is collapsed to a suitable two-element path. It is to ensure that the desired connection may be made between each $U_{i_{s}}^{\prime}$ and its "natural" image $U_{s}$, that parity conditions feature in the order definition.

We define $\zeta\left(x_{i}^{\prime}\right)$ for $i=1, \ldots, m$ as follows:

- for $i_{s-1}<i \leq i_{s}$ (setting $i_{0}=0$ ),

$$
x_{i}^{\prime} \mapsto \begin{cases}x_{s}, & \text { if }\left(i-i_{s-1}\right) \text { is even } \\ x_{s+1}, & \text { if }\left(i-i_{s-1}\right) \text { is odd; }\end{cases}
$$

- for $i>i_{k}$

$$
x_{i}^{\prime} \mapsto \begin{cases}x_{k}, & \text { if }\left(i-i_{k}\right) \text { is even } \\ x_{k+1}, & \text { if }\left(i-i_{k}\right) \text { is odd. }\end{cases}
$$

Next, we define $\zeta\left(y_{j}^{\prime}\right)$ for $j=1, \ldots, n$ : 
- for $j_{s-1}<j \leq j_{s}$ (setting $\left.j_{0}=0\right), y_{j}^{\prime} \mapsto y_{s}$;

- for $j>j_{l}, y_{j}^{\prime} \mapsto y_{l}$.

Finally, we define $\zeta$ on $U_{i}^{\prime}(i=1, \ldots, m)$ and on $V_{j}^{\prime}(j=1, \ldots, n)$ :

- if $i=i_{s}, U_{i}^{\prime}$ is mapped onto $U_{s}$ via $\mu_{s}$;

- otherwise, $U_{i}^{\prime}$ is mapped onto an edge emanating from $\zeta\left(x_{i}^{\prime}\right)$, as guaranteed by Lemma 3.9;

- if $j=j_{s}, V_{j}^{\prime}$ is mapped onto $V_{s}$ via $v_{s}$;

- otherwise, $V_{j}^{\prime}$ is mapped onto an edge emanating from $\zeta\left(y_{j}^{\prime}\right)$, as guaranteed by Lemma 3.9.

A routine check shows that $\zeta$ is an epimorphism.

Since $\tau$ is order-preserving and $\mathcal{P}$ is $\mathrm{PWO}$, it follows that $\tau(\mathcal{P})$ is $\mathrm{PWO}$, and we have seen that $\tau(\mathcal{P})$ contains $\operatorname{Av}(S)$, proving the lemma.

We can now proceed to stating and proving the main results of this section:

Theorem 3.11. Let $\mathcal{T}^{\bullet}$ be the class of all irreflexive rooted trees. Then $\mathcal{T}^{\bullet}$ is $P W O$ with respect to $O_{\mathrm{H}}$.

Proof. Suppose $T_{1}, T_{2}, \ldots$ forms an infinite antichain of irreflexive rooted trees. Without loss, assume that $T_{1}$ is non-trivial. Then $T_{2}, T_{3}, \ldots$ is an infinite antichain in $\operatorname{Av}\left(T_{1}\right)$, in contradiction to Lemma 3.10.

Corollary 3.12. Let $\mathcal{C}$ be the class of irreflexive trees. Then $\mathcal{C}$ is $P W O$ with respect to $O_{\mathrm{H}}$.

Proof. Let $T_{1}, T_{2}, \ldots$ be an infinite collection of irreflexive trees. In each $T_{i}$, pick an arbitrary vertex $r_{i}$. Denote by $T_{i}^{*}$ the rooted tree obtained from $T_{i}$ by designating $r_{i}$ as the root. By Theorem 3.11, for some $i \neq j$ there exists an epimorphism of irreflexive rooted trees $\phi: T_{i}^{*} \rightarrow T_{j}^{*}$. Thus $\phi$ maps edges of $T_{i}$ to edges of $T_{j}$ and maps $r_{i}$ to $r_{j}$. In particular, $\phi$ is also an epimorphism of non-rooted trees, $T_{i} \rightarrow T_{j}$, and the corollary follows.

Corollary 3.13. Let $\mathcal{C}$ be the class of reflexive trees. Then $\mathcal{C}$ is $P W O$ with respect to $O_{\mathrm{H}}$.

Proof. Let $T_{1}, T_{2}, \ldots$ be an infinite collection of reflexive trees. Let $T_{i}^{\prime}$ be the irreflexive tree obtained by removing the loops at each vertex from $T_{i}$. By Corollary 3.12, there is an epimorphism $\phi: T_{i}^{\prime} \rightarrow T_{j}^{\prime}$ for some $i \neq j$. Thus $\phi$ maps non-loop edges of $T_{i}$ to non-loop edges of $T_{j}$ and, since $\phi$ trivially maps loops of $T_{i}$ to loops of $T_{j}$, it follows that $\phi$ is also an epimorphism $T_{i} \rightarrow T_{j}$.

\subsection{Equivalence relations}

Let $\mathcal{A}=(A, R)$ and $\mathcal{B}=(B, S)$ be equivalence relations. Let $A_{1}, \ldots, A_{k}$ be the equivalence classes of $R$, and $B_{1}, \ldots, B_{l}$ be the equivalence classes of $S$. As we have seen in Subsection 2.2, a mapping $\phi: A \rightarrow B$ is

- a homomorphism if $(\forall i)(\exists j)\left(\phi\left(A_{i}\right) \subseteq B_{j}\right)$;

- a strong homomorphism if it is a homomorphism and for any $y_{1}, y_{2} \in B_{j} \cap \operatorname{im} \phi$ we have

$$
(\exists i)\left(\exists x_{1}, x_{2} \in A_{i}\right)\left(\phi\left(x_{1}\right)=y_{1}, \phi\left(x_{2}\right)=y_{2}\right) ;
$$


- an M-strong homomorphism if it is a homomorphism and

$$
\phi\left(A_{i_{1}}\right), \phi\left(A_{i_{2}}\right) \subseteq B_{j} \Rightarrow i_{1}=i_{2} .
$$

From the above definitions, we have the following descriptions of $O_{\mathrm{H}}, O_{\mathrm{IH}}$ and $\mathrm{O}_{\mathrm{MH}}$ :

(E1) $\mathcal{A} \preceq_{\mathrm{H}} \mathcal{B} \Leftrightarrow \exists \phi: A \rightarrow B$ such that every $B_{j}$ is a union of some $\phi\left(A_{i}\right)$;

(E2) $\mathcal{A} \preceq_{\mathrm{IH}} \mathcal{B} \Leftrightarrow \exists \phi: A \rightarrow B$ such that for any $y_{1}, y_{2} \in B_{j}, \exists i: y_{1}, y_{2} \in \phi\left(A_{i}\right)$;

(E3) $\mathcal{A} \preceq_{\text {MH }} \mathcal{B} \Leftrightarrow k=l$ and there is a permutation $j_{1}, \ldots, j_{k}$ of $1, \ldots, k$ such that $\phi\left(A_{i}\right)=B_{j_{i}}$.

We prove that the class of equivalence relations is $\mathrm{PWO}$ under $\mathrm{O}_{\mathrm{IH}}$, which implies that it is PWO under $O_{\mathrm{H}}$.

Lemma 3.14. Let $\mathcal{A}=(A, R)$ and $\mathcal{B}=(B, S)$ be two equivalence relations. If $R$ has $k$ non-singleton equivalence classes, $|B|=n$ and $k \geq n^{2}$ then $\mathcal{B} \preceq_{\mathrm{IH}} \mathcal{A}$.

Proof. Let $A_{1}, \ldots, A_{k}$ be the non-singleton equivalence classes of $R$. Since

$$
k \geq n^{2}=|B|^{2} \geq|S|
$$

there exists a surjection $f:\left\{A_{1}, \ldots, A_{k}\right\} \rightarrow S$. Since $\left|A_{i}\right| \geq 2$, there exists a surjection $\phi_{i}: A_{i} \rightarrow$ $\left\{b^{\prime}, b^{\prime \prime}\right\}$ where $f\left(A_{i}\right)=\left(b^{\prime}, b^{\prime \prime}\right) \in S$. Let $\phi^{\prime}: A \backslash\left(\bigcup_{i=1}^{k} A_{i}\right) \rightarrow B$ be arbitrary. The mapping $\phi=\left(\bigcup_{i=1}^{k} \phi_{i}\right) \cup \phi^{\prime}$ is an epimorphism, and indeed strong by (E2).

Theorem 3.15. The class of equivalence relations is $P W O$ with respect to $O_{\mathrm{IH}}$ (and hence also with respect to $\mathrm{O}_{\mathrm{H}}$ ).

Proof. Let $\mathcal{A}_{s}=\left(A_{s}, R_{s}\right)(s=1,2, \ldots)$ be an infinite collection of equivalence relations. Let $c_{s}$ be the number of non-singleton equivalence classes of $R_{s}$. We distinguish two cases.

Case 1: $\left\{c_{s}: s \in \mathbb{N}\right\}$ is unbounded. Let $s \in \mathbb{N}$ be such that $c_{s} \geq\left|A_{1}\right|^{2}$. By Lemma 3.14, we have $\mathcal{A}_{1} \preceq_{\mathrm{IH}} \mathcal{A}_{s}$.

Case 2: $\left\{c_{s}: s \in \mathbb{N}\right\}$ is bounded. Without loss we may assume that $c_{1}=c_{2}=\cdots=c$. So, each $R_{s}$ has precisely $c$ non-singleton classes, say $A_{s, 1}, A_{s, 2} \ldots, A_{s, c}$, and, say, $t_{s}$ singleton ones. Consider the collection of $(c+1)$-tuples

$$
\left\{\left(\left|A_{s, 1}\right|, \ldots,\left|A_{s, c}\right|, t_{s}\right): s=1,2, \ldots\right\} .
$$

By Higman's Theorem ([7]), this is PWO with respect to tuple ordering. Hence there are distinct $u, v \in \mathbb{N}$ such that

$$
\left(\left|A_{u, 1}\right|, \ldots,\left|A_{u, c}\right|, t_{u}\right) \leq\left(\left|A_{v, 1}\right|, \ldots,\left|A_{v, c}\right|, t_{v}\right) .
$$

Hence there are onto mappings $\phi_{d}: A_{v, d} \rightarrow A_{u, d}$ and $\phi^{\prime}: A_{v} \backslash\left(\bigcup_{d=1}^{c} A_{v, d}\right) \rightarrow A_{u} \backslash\left(\bigcup_{d=1}^{c} A_{u, d}\right)$. Their union $\phi:\left(\bigcup_{d=1}^{c} \phi_{d}\right) \cup \phi^{\prime}$ is an epimorphism, which is strong by (E2).

Theorem 3.16. Let $\mathcal{C}$ be the class of equivalence relations. Then $\mathcal{C}$ is not $P W O$ with respect to $O_{\mathrm{MH}}$.

Proof. Let $\mathcal{A}_{n}=\left(A_{n}, R_{n}\right)$ where $R_{n}$ has precisely $n$ equivalence classes. Then $\left(\mathcal{A}_{1}, \mathcal{A}_{2}, \ldots\right)$ is an antichain by (E3). 


\subsection{Linear orders, words and permutations}

Proposition 3.17. The class of linear orders is $P W O$ with respect to $O_{\mathrm{H}}$ and $\mathrm{O}_{\mathrm{IH}}$, but not with respect to $\mathrm{O}_{\mathrm{MH}}$.

Proof. A chain may be mapped onto any shorter chain under $\mathrm{O}_{\mathrm{H}}$; the other cases follow from Table 1.

Proposition 3.18. Neither of the following classes is $P W O$ with respect to $O_{\mathrm{H}}$ :

- the class of words over a non-singleton alphabet;

- the class of permutations.

Proof. In the word case, an infinite antichain is given by any infinite collection of words with no consecutive occurrences of any letter, for example $(a b)^{n}, n \in \mathbb{N}$. Likewise, in the permutation case, any infinite set of permutations without non-trivial intervals (also known as simple permutations) would suffice; there are many such examples in the literature (see [1] and [2]).

\section{Joint Preimage Property}

Here, we address the question: for a given class $\mathcal{C}$ under a given homomorphic image order, does $\mathcal{C}$ possess the joint preimage property (JPP)? Unlike in the previous section, there is no single consistent division in behaviour between M-strong order and the other two; the orders are "grouped" differently as we move between different classes of structures. From the tables, the following patterns are observable: the answer to the JPP question is negative in all cases for the M-strong homomorphic image order, approximately half-and-half for the strong homomorphic image order, and largely positive for the homomorphic image order.

We first consider classes of structure which do not possess the JPP property under any of the three orders; in each case, it is sufficient to prove the assertion for $O_{\mathrm{H}}$.

Theorem 4.1. The class of irreflexive trees does not possess the JPP under any of the homomorphic image orderings.

Proof. This follows from the fact that the single-vertex tree is not the homomorphic image of any tree other than itself.

We observe that the class of non-trivial irreflexive trees would possess JPP, via a proof similar to that for reflexive trees (Theorem 4.5).

Theorem 4.2. The class of tournaments (irreflexive or reflexive) does not possess the JPP under any of the homomorphic image orderings.

Proof. The irreflexive case is trivial, since irreflexive tournaments are minimal. We consider the reflexive case. Let $A=T_{3}$ and $B=T_{5}$ from Proposition 3.5. So $A$ is the directed triangle $a \rightarrow b \rightarrow c \rightarrow a$, and neither of $A$ nor $B$ has proper non-trivial homomorphic image.

Suppose $A$ and $B$ have a joint preimage $C$, say with $\phi: C \rightarrow A$ and $\psi: C \rightarrow B$. For every $x \in A$, pick a preimage $x^{\prime} \in C$ under $\phi$. Then $A^{\prime}=\left\{a^{\prime}, b^{\prime}, c^{\prime}\right\}$ is a tournament isomorphic to $A$. Likewise, there exists a tournament $B^{\prime}=\left\{x^{\prime}: x \in B\right\}$ inside $C$ which is isomorphically mapped onto $B$ under $\psi$.

Since $B$ has no proper non-trivial homomorphic image and $|B|>|A|$, it follows that $\phi\left(B^{\prime}\right)$ is a single point of $A$, say $\phi\left(B^{\prime}\right)=\{a\}$. From $a^{\prime} \rightarrow b^{\prime}$, we have that $x^{\prime} \rightarrow b^{\prime}$ for all $x^{\prime} \in B^{\prime}$. Suppose $\psi\left(b^{\prime}\right)=y \in B$. Let $z \in B$ be such that $y \rightarrow z$ and $y \neq z$. Then $b^{\prime} \rightarrow z^{\prime}$, a contradiction. 
Theorem 4.3. Neither of the following possesses the JPP under any of the homomorphic image orderings: (i) the class of words over a non-singleton alphabet, (ii) permutations.

Proof. In the word case, a counterexample is given by taking any two distinct letters from the alphabet, and in the permutation case by taking 12 and 21.

Next, we consider those classes which possess the JPP under $O_{\mathrm{H}}$, but not under the two stronger orders.

Theorem 4.4. The following classes possess the JPP under $\mathrm{O}_{\mathrm{H}}$ but not under $\mathrm{O}_{\mathrm{IH}}$ or $\mathrm{O}_{\mathrm{MH}}$ : (i) digraphs, (ii) irreflexive graphs, (iii) irreflexive posets.

Proof. To prove that digraphs possess the JPP under $\mathrm{O}_{\mathrm{H}}$, it is sufficient to note that an empty graph has any other smaller digraph as a homomorphic image. On the other hand, any preimage of an empty digraph is empty, while any strong preimage of a non-empty digraph is non-empty. Hence, such a pair of graphs cannot join. To complete the proof, note that empty digraphs belong to all three listed classes.

Finally, we consider those classes which possess JPP under $O_{\mathrm{IH}}$ (and hence $O_{\mathrm{H}}$ ) but not under $\mathrm{O}_{\mathrm{MH}}$.

Theorem 4.5. The following classes possess the JPP under $\mathrm{O}_{\mathrm{H}}$ and $\mathrm{O}_{\mathrm{IH}}$, but not $\mathrm{O}_{\mathrm{MH}}$ : (i) reflexive graphs, (ii) reflexive trees, (iii) reflexive posets, (iv) equivalence relations, (v) linear orders.

Proof. Let $\mathcal{C}$ be any of the above classes, and let $A, B \in \mathcal{C}$. Take $C$ to be:

- the disjoint union of $A$ and $B$ in cases (i), (iii), (iv);

- a union of $A$ and $B$ with a single-vertex intersection in case (ii);

- the order sum of $A$ and $B$ in case (v).

Note that $C \in \mathcal{C}$ in each case. Furthermore, both $A$ and $B$ are strong homomorphic images of $C$. For instance, we can map a copy of $A$ inside $C$ bijectively onto $A$, and map the copy of $B$ to an appropriate single point of $A$. Hence $\mathcal{C}$ has the JPP under $O_{\mathrm{IH}}$.

We now prove that $\mathcal{C}$ does not possess the JPP under $O_{\mathrm{MH}}$. In cases (ii), (iii), (v) this is due to minimality; see Table 1. For cases (i), (iv) note that every M-strong preimage of a complete digraph is necessarily complete, while an M-strong preimage of the (reflexive) empty digraph is a disjoint union of complete digraphs. Hence two such digraphs do not join.

\section{Dual amalgamation property}

We now move on to our final question: for a given class $\mathcal{C}$ under a given homomorphic image order, does $\mathcal{C}$ possess the dual amalgamation property $(D A P)$ ? In this context, the arguments necessary for each of the three types of order are rather different in nature. This is due to the fact that epimorphisms feature under the assumptions for this property, and therefore, say, DAP for the $\mathrm{M}$-induced order is not a specialisation of DAP for the induced order. Despite this, it transpires that the answer to the DAP question is positive for most classes and orders.

We begin with the following lemma:

Lemma 5.1. The Dual Amalgamation Property holds for unstructured sets with respect to surjections. 
Proof. Let $A, B, C$ be sets and let $f: B \rightarrow A, g: C \rightarrow A$ be surjections. We will prove the existence of a set $D$ and epimorphisms $h: D \rightarrow B$ and $k: D \rightarrow C$ such that $f h=g k$. For each $a \in A$, consider the sets $f^{-1}(a) \subseteq B$ and $g^{-1}(a) \subseteq C$; both are non-empty. Let $D_{a}$ be pairwise disjoint sets such that $\left|D_{a}\right| \geq \max \left(\left|f^{-1}(a)\right|,\left|g^{-1}(a)\right|\right)$. Let $h_{a}: D_{a} \rightarrow f^{-1}(a)$ and $k_{a}: D_{a} \rightarrow g^{-1}(a)$ be arbitrary surjections. Let $D=\bigcup_{a \in A} D_{a}, h=\bigcup_{a \in A} h_{a}$ and $k=\bigcup_{a \in A} k_{a}$. Since $\operatorname{im}\left(h_{a}\right)=f^{-1}(a)$ and $\bigcup_{a \in A} f^{-1}(a)=B$, we have that $h$ is onto. Analogously, $k$ is onto. Let $d \in D$, say $d \in D_{a}$. Then $h(d)=h_{a}(d) \in f^{-1}(a)$ and $k(d)=k_{a}(d) \in g^{-1}(a)$. Hence $f h(d)=a=g k(d)$, as required.

Theorem 5.2. The following classes possess the DAP with respect to $\mathrm{O}_{\mathrm{H}}$ : (i) digraphs, (ii) reflexive graphs, (iii) irreflexive graphs, (iv) reflexive posets, (v) irreflexive posets, (vi) irreflexive tournaments, (vii) equivalence relations, (viii) linear orders, (ix) words.

Proof. Case (vi) is immediate because $O_{\mathrm{MH}}$ reduces to isomorphism (see Table 1).

For cases (i), (ii), (iii), (iv), (v), (vii) and (viii), we apply Lemma 5.1. For example, given digraphs $A, B, C$ and epimorphisms $f: B \rightarrow A, g: C \rightarrow A$, apply the process in the proof of Lemma 5.1 to the vertices of $A, B$ and $C$ to obtain the vertices of $D=\bigcup_{a \in A} D_{a}$, and take $D$ to be the empty digraph on these vertices. This construction may then be appropriately adapted, with "empty digraph" replaced by the appropriate analogous structure: reflexive/irreflexive empty graph in cases (ii) and (iii), reflexive/irreflexive antichain in cases (iv) and (v), the diagonal relation in case (vii) and the order-sum of chains $D_{a}$ in case (viii).

For case (ix), let $A, B, C$ be words and $f: B \rightarrow A, g: C \rightarrow A$ be epimorphisms. Suppose $A=x_{1}^{\alpha_{1}} x_{2}^{\alpha_{2}} \ldots x_{k}^{\alpha_{k}}$ where $x_{1}, \ldots, x_{k} \in X$ and $x_{i} \neq x_{i+1}$. The existence of homomorphisms $f$ and $g$ implies that $B$ and $C$ have the same "form" as $A$ but with different exponents; more precisely $B=x_{1}^{\beta_{1}} \ldots x_{k}^{\beta_{k}}$ and $C=x_{1}^{\gamma_{1}} \ldots x_{k}^{\gamma_{k}}$ where $\beta_{i}, \gamma_{i} \geq \alpha_{i}$. Then $D=x_{1}^{\delta_{1}} \ldots x_{k}^{\delta_{k}}$ where $\delta_{i}=\max \left(\beta_{i}, \gamma_{i}\right)$, with the obvious mappings $h: D \rightarrow B, k: D \rightarrow C$, satisfies the definition of DAP.

Theorem 5.3. The following classes do not possess the DAP with respect to $\mathrm{O}_{\mathrm{H}}$ : (i) reflexive trees, (ii) irreflexive trees, (iii) reflexive tournaments, (iv) permutations.

Proof. Cases (iii) and (iv) follow since, for each class, the JPP does not hold and the trivial structure is a homomorphic image of each member.

For cases (i) and (ii), let $A$ be a star with four vertices $\{1,2,3,4\}$ and edges $(1,2),(1,3)$ and $(1,4)$. Let $B=\left\{b_{1}, \ldots, b_{11}\right\}$ and $C=\left\{c_{1}, \ldots, c_{11}\right\}$ be paths of length 11 , and let $f: B \rightarrow A$ and $g: C \rightarrow A$ be the epimorphisms given by:

$$
\begin{aligned}
f & =\left(\begin{array}{ccccccccccc}
b_{1} & b_{2} & b_{3} & b_{4} & b_{5} & b_{6} & b_{7} & b_{8} & b_{9} & b_{10} & b_{11} \\
1 & 4 & 1 & 3 & 1 & 2 & 1 & 3 & 1 & 4 & 1
\end{array}\right) \\
g & =\left(\begin{array}{ccccccccccc}
c_{1} & c_{2} & c_{3} & c_{4} & c_{5} & c_{6} & c_{7} & c_{8} & c_{9} & c_{10} & c_{11} \\
1 & 3 & 1 & 4 & 1 & 2 & 1 & 4 & 1 & 3 & 1
\end{array}\right) .
\end{aligned}
$$

Suppose there exists a tree $D$ and epimorphisms $h: D \rightarrow B$ and $k: D \rightarrow C$ such that $f h=g k$. Let $p, q \in D$ be such that $h(p)=b_{4}, h(q)=b_{6}$ and the unique path $p=d_{1}, d_{2}, \ldots, d_{k}=q$ is shortest possible. Then of course $h\left(d_{i}\right) \notin\{p, q\}$ for $i \neq 1, k$. It then follows that in fact $h\left(d_{i}\right)=b_{5}$ for $i=2, \ldots, k-1$. Hence we have $f h\left(\left\{d_{1}, \ldots, d_{k}\right\}\right)=\{3,1,2\}$. (In the irreflexive case we must have $k=3$.) Consider now the image of the path $d_{1}, \ldots, d_{k}$ in $C$. Its vertices form a contiguous segment $c_{l}, \ldots, c_{m}$. From the definition of $g$ we see that no such contiguous segment is mapped precisely onto the set $\{3,1,2\}$. Hence $f h \neq g k$.

We next consider the strong homomorphism definition. 
Theorem 5.4. The following classes possess the DAP with respect to $O_{\mathrm{IH}}$ : (i) digraphs, (ii) reflexive graphs, (iii) irreflexive graphs, (iv) reflexive posets, (v) irreflexive posets, (vi) irreflexive tournaments, (vii) equivalence relations, (viii) linear orders, (ix) words.

Proof. For case (i), let $A, B, C$ be digraphs and let $f: B \rightarrow A, g: C \rightarrow A$ be strong epimorphisms. We will prove the existence of a digraph $D$ and epimorphisms $h: D \rightarrow B$ and $k: D \rightarrow C$ such that $f h=g k$.

For every edge $\alpha=\left(a_{1}, a_{2}\right)$ in $E(A)$, there are edges

$$
\left(F\left(\alpha, a_{1}\right), F\left(\alpha, a_{2}\right)\right) \in E(B) \text { and }\left(G\left(\alpha, a_{1}\right), G\left(\alpha, a_{2}\right)\right) \in E(C)
$$

such that

$$
f\left(F\left(\alpha, a_{1}\right)\right)=a_{1}, f\left(F\left(\alpha, a_{2}\right)\right)=a_{2}, g\left(G\left(\alpha, a_{1}\right)\right)=a_{1}, g\left(G\left(\alpha, a_{2}\right)\right)=a_{2} .
$$

We construct $D$ as follows. For every edge $\beta=\left(b_{1}, b_{2}\right) \in E(B)$, let $D_{B, \beta}=\left\{d_{1, \beta}, d_{2, \beta}\right\}$, and for every edge $\gamma=\left(c_{1}, c_{2}\right) \in E(C)$, let $D_{C, \gamma}=\left\{d_{1, \gamma}, d_{2, \gamma}\right\}$. Now let $D=D_{B} \cup D_{C}$ where $D_{B}=\bigcup_{\beta \in E(B)} D_{B, \beta}$ and $D_{C}=\bigcup_{\gamma \in E(C)} D_{C, \gamma}$ and let the edges be

$$
E(D)=\left\{\left(d_{1, \beta}, d_{2, \beta}\right): \beta \in E(B)\right\} \cup\left\{\left(d_{1, \gamma}, d_{2, \gamma}\right): \gamma \in E(C)\right\}
$$

We now define homomorphisms $h: D \rightarrow B$ and $k: D \rightarrow C$ as follows. For $x=d_{i, \beta} \in D_{B, \beta}$, with $\beta=\left(b_{1}, b_{2}\right)$, we let $a_{1}=f\left(b_{1}\right), a_{2}=f\left(b_{2}\right)$, note that $\alpha=\left(a_{1}, a_{2}\right) \in E(A)$, and define

$$
h(x)=b_{i}, k(x)=G\left(\alpha, a_{i}\right) .
$$

Analogously, for $x=d_{i, \gamma} \in D_{C, \gamma}$ with $\gamma=\left(c_{1}, c_{2}\right)$, we let $a_{1}=g\left(c_{1}\right), a_{2}=g\left(c_{2}\right)$, note that $\alpha=\left(a_{1}, a_{2}\right) \in E(A)$, and define

$$
h(x)=F\left(\alpha, a_{i}\right), k(x)=c_{i} .
$$

From this definition it is clear that $h$ maps edges of $D$ onto the edges of $B$, and does so bijectively when restricted to $D_{B}$. Hence $h$, and dually $k$, are strong epimorphisms.

We claim that $f h=g k$. Indeed, let $x \in D$, and without loss, assume $x=d_{1, \beta}$. With the notation as above

$$
f h(x)=f h\left(d_{1, \beta}\right)=f\left(b_{1}\right)=a_{1}=g\left(G\left(\alpha, a_{1}\right)\right)=g k\left(d_{1, \beta}\right)=g k(x),
$$

as required.

We may adapt this proof for cases (ii), (iii), (iv), (v) and (vii); for irreflexive graphs and posets the argument follows the digraph proof precisely. For reflexive graphs and posets and for equivalence relations, loops at every vertex must be added in the construction.

Cases (vi), (viii) and (ix) all follow from Theorem 5.2 and the fact that all homomorphisms are strong (see Table 1$)$.

Theorem 5.5. The following classes do not possess the DAP with respect to $O_{\mathrm{IH}}$ : (i) reflexive trees, (ii) irreflexive trees, (iii) reflexive tournaments, (iv) permutations.

Proof. The proof of Theorem 5.3 remains valid because all homomorphisms are strong. 
In the M-strong case, it turns out that the dual amalgamation property holds for all-but-one of the types of structure under consideration, but for reasons that are rather different to those we have seen so far. Essentially, in order for $A, B$ and $C$ to possess surjective homomorphisms $f: B \rightarrow A$ and $g: C \rightarrow A$ under the M-strong definition, the situation is already very constrained, with the structure of $B$ and $C$ being tightly controlled by that of $A$. This allows the construction of a suitable $D$, also determined by $A$. It is worth recalling that the Joint Preimage Property does not hold for any of the classes under $O_{\mathrm{MH}}$.

Theorem 5.6. All the following classes possess DAP with respect to $\mathrm{O}_{\mathrm{MH}}$ : (i) digraphs, (ii) reflexive graphs, (iii) irreflexive graphs, (iv) reflexive trees, (v) reflexive posets, (vi) irreflexive posets, (vii) reflexive tournaments, (viii) irreflexive tournaments, (ix) equivalence relations, $(x)$ linear orders, $(x i)$ permutations, (xii) words.

Proof. For case (i), let $A, B, C$ be digraphs and let $f: B \rightarrow A, g: C \rightarrow A$ be M-strong epimorphisms. We will prove the existence of a digraph $D$ and M-strong epimorphisms $h: D \rightarrow B$ and $k: D \rightarrow C$ such that $f h=g k$.

For each $a \in A$, define $B(a)=f^{-1}(a)$ and $C(a)=g^{-1}(a)$. Since $f$ and $g$ are onto, we have $B=\bigcup_{a \in A} B(a)$ and $C=\bigcup_{a \in A} C(a)$. Let $D(a)(a \in A)$ be pairwise disjoint sets with $|D(a)| \geq$ $\max (|B(a)|,|C(a)|)$. Let $h_{a}: D(a) \rightarrow B(a)$ and $k_{a}: D(a) \rightarrow C(a)$ be arbitrary surjections. Let $D=\bigcup_{a \in A} D(a), h=\bigcup_{a \in A} h_{a}$ and $k=\bigcup_{a \in A} k_{a}$.

Define a digraph structure on $D$ as follows: for $d_{1}, d_{2} \in D$ with $d_{i} \in D\left(a_{i}\right)\left(a_{1}, a_{2} \in A\right)$, we let

$$
\left(d_{1}, d_{2}\right) \in E(D) \Leftrightarrow\left(a_{1}, a_{2}\right) \in E(A) .
$$

Since $D=\bigcup_{a \in A} D(a), B=\bigcup_{a \in A} B(a)$ and $h_{a}: D(a) \rightarrow B(a)$ is onto, it follows that $h: D \rightarrow B$ is onto. For arbitrary $d_{1}, d_{2} \in D$, with $d_{i} \in D\left(a_{i}\right)$, let $b_{i}=h\left(d_{i}\right) \in B\left(a_{i}\right)$, and then

$$
\begin{aligned}
\left(d_{1}, d_{2}\right) \in E(D) & \Leftrightarrow\left(a_{1}, a_{2}\right) \in E(A) & & \text { (by }(1)) \\
& \Leftrightarrow\left(f\left(b_{1}\right), f\left(b_{2}\right)\right) \in E(A) & & \text { (since } \left.B_{i}=f^{-1}\left(a_{i}\right)\right) \\
& \Leftrightarrow\left(b_{1}, b_{2}\right) \in E(B) & & \text { (since } f \text { is M-strong) } \\
& \Leftrightarrow\left(h\left(d_{1}\right), h\left(d_{2}\right)\right) \in E(B) & & \text { (since } \left.b_{i}=h\left(d_{i}\right)\right)
\end{aligned}
$$

Hence $h$ is an M-strong homomorphism, and so is $k$ by an analogous argument.

Finally, we check that $f h=g k$. Let $d \in D$, say $d \in D(a)$. Then $h(d)=h_{a}(d) \in B(a)$ and so $f h(d)=a$. By symmetry, $g k(d)=a$ and hence $f h=g k$.

The digraph construction may also be used to establish the result in cases (ii), (iii), (vi) and (ix). Cases (v), (vii), (viii), (x), (xi) and (xii) are trivial, since $O_{\mathrm{MH}}$ reduces to isomorphism; see Table 1. Case (iv) follows similarly from Lemma 2.6.

Theorem 5.7. The class of irreflexive trees does not possess DAP with respect to $\mathrm{O}_{\mathrm{MH}}$.

Proof. Let $A, B$ and $C$ be the paths $1-2,3-4-5$ and $6-7-8$ respectively. Let $f: B \rightarrow A$ and $g: C \rightarrow A$ be M-strong homomomorphisms defined by

$$
f=\left(\begin{array}{lll}
3 & 4 & 5 \\
2 & 1 & 2
\end{array}\right), g=\left(\begin{array}{lll}
6 & 7 & 8 \\
1 & 2 & 1
\end{array}\right) \text {. }
$$

Suppose there exists a tree $D$ and M-strong homomorphisms $h: D \rightarrow B, k: D \rightarrow C$ such that $f h=g k$. Note that $D$ is a disjoint union $D_{1} \cup D_{2}$, where $D_{i}=(f h)^{-1}(i)=(g k)^{-1}(i)$. Since 1 and 
2 are connected in $A$, and $f h=g k$ is an M-strong homomorphism, it follows that every vertex from $D_{1}$ is connected to every vertex from $D_{2}$. Furthermore

$$
D_{1}=(g k)^{-1}(1)=k^{-1} g^{-1}(1)=k^{-1}\{6,8\},
$$

implying that $\left|D_{1}\right| \geq 2$ and, likewise, $\left|D_{2}\right| \geq 2$. But then the edges between $D_{1}$ and $D_{2}$ must form cycles, contradicting $D$ being a tree.

\section{Concluding remarks}

In this paper we have begun an investigation into the homomorphic image order, structured around the three topics of partial well-order, joint preimage property and dual amalgamation property. There are of course many avenues open for future investigation. The antichain results presented here give a fairly uniform picture; this undoubtedly changes in the presence of avoidance restrictions. So one possible research direction is: in each of our contexts, classify partially well-ordered classes defined by a small number of small obstructions. In the setting of substructure order, the Joint Embedding Property and the Amalgamation Property lead to the notions of atomic and homogeneous structures. It is natural to ask whether our orders $O_{\mathrm{H}}, O_{\mathrm{IH}}$ and $O_{\mathrm{MH}}$ admit of a similar treatment, and whether this would lead to interesting combinatorial objects with universal properties.

Acknowledgement. The authors are grateful to an anonymous referee for a careful reading of the paper and helpful comments.

\section{References}

[1] M. Albert and M. Atkinson, Simple permutations and pattern restricted permutations, Discrete Math. 300 (2005), 1-15.

[2] R. Brignall, A survey of simple permutations, Permutation patterns, 41-65, London Math. Soc. Lecture Note Ser. 376, Cambridge Univ. Press, Cambridge, 2010.

[3] P.J. Cameron and P.A. Kazanidis, Cores of symmetric graphs, J. Austral. Math. Soc. 85 (2008), 145-154.

[4] G. Cherlin, Forbidden substructures and combinatorial dichotomies: WQO and universality, Discrete Math. 311 (2011), 1543-1584.

[5] J. Foniok, J. Nešetřil and C. Tardif, Generalised dualities and maximal finite antichains in the homomorphism order of relational structures, Europ. J. Combinat. 29 (2008), 881-899.

[6] P. Hell and J. Nešetřil, Graphs and Homomorphisms, Oxford Lecture Series in Mathematics and its applications 28, Oxford University Press, Oxford, 2004.

[7] G. Higman, Ordering by divisibility in abstract algebras, Proc. London Math. Soc. 2 (1952), 326-336.

[8] W. Hodges, Model theory, Cambridge University Press, 1993.

[9] J.B. Kruskal, The theory of well-quasi-ordering: A frequently discovered concept, J. Combinat. Theory Ser. A 13 (1972), 297-305. 
[10] L. Kwuida and E. Lehtonen, On the homomorphism order of labeled posets, Order 28 (2011), 251-265.

[11] J. Nešetřil and A. Pultr, On classes of relations and graphs determined by subobjects and factorobjects, Discrete Math. 22 (1978), 287-300. 
Table 2: Summary of results for the homomorphic image order

\begin{tabular}{lccc}
\hline \hline Class $\mathcal{C}$ & PWO & JPP & DAP \\
\hline Digraphs & No: Theorem 3.1 & Yes: Theorem 4.4 & Yes: Theorem 5.2 \\
Graphs(reflexive) & No: Theorem 3.3 & Yes: Theorem 4.5 & Yes: Theorem 5.2 \\
Graphs (irreflexive) & No: Theorem 3.2 & Yes: Theorem 4.4 & Yes: Theorem 5.2 \\
Trees (reflexive) & Yes: Corollary 3.13 & Yes: Theorem 4.5 & No: Theorem 5.3 \\
Trees (irreflexive) & Yes: Corollary 3.12 & No: Theorem 4.1 & No: Theorem 5.3 \\
Posets (reflexive) & No: Theorem 3.8 & Yes: Theorem 4.5 & Yes: Theorem 5.2 \\
Posets (irreflexive) & No: Theorem 3.7 & Yes: Theorem 4.4 & Yes: Theorem 5.2 \\
Tournaments (reflexive) & No: Theorem 3.6 & No: Theorem 4.2 & No: Theorem 5.3 \\
Tournaments (irreflexive) & No: Theorem 3.6 & No: Theorem 4.2 & Yes: Theorem 5.2 \\
Equivalence relations & Yes: Theorem 3.15 & Yes: Theorem 4.5 & Yes: Theorem 5.2 \\
Linear orders & Yes: Proposition 3.17 & Yes: Theorem 4.5 & Yes: Theorem 5.2 \\
Permutations & No: Proposition 3.18 & No: Theorem 4.3 & No: Theorem 5.3 \\
Words & No: Proposition 3.18 & No: Theorem 4.3 & Yes: Theorem 5.2 \\
\hline
\end{tabular}

Table 3: Summary of results for the strong homomorphic image order

\begin{tabular}{lccc}
\hline \hline Class $\mathcal{C}$ & PWO & JPP & DAP \\
\hline Digraphs & No: Theorem 3.1 & No: Theorem 4.4 & Yes: Theorem 5.4 \\
Graphs(reflexive) & No: Theorem 3.3 & Yes: Theorem 4.5 & Yes: Theorem 5.4 \\
Graphs (irreflexive) & No: Theorem 3.2 & No: Theorem 4.4 & Yes: Theorem 5.4 \\
Trees (reflexive) & Yes: Corollary 3.13 & Yes: Theorem 4.5 & No: Theorem 5.5 \\
Trees (irreflexive) & Yes: Corollary 3.12 & No: Theorem 4.1 & No: Theorem 5.5 \\
Posets (reflexive) & No: Theorem 3.8 & Yes: Theorem 4.5 & Yes: Theorem 5.4 \\
Posets (irreflexive) & No: Theorem 3.7 & No: Theorem 4.4 & Yes: Theorem 5.4 \\
Tournaments (reflexive) & No: Theorem 3.6 & No: Theorem 4.2 & No: Theorem 5.5 \\
Tournaments (irreflexive) & No: Theorem 3.6 & No: Theorem 4.2 & Yes: Theorem 5.4 \\
Equivalence relations & Yes: Theorem 3.15 & Yes: Theorem 4.5 & Yes: Theorem 5.4 \\
Linear orders & Yes: Proposition 3.17 & Yes: Theorem 4.5 & Yes: Theorem 5.4 \\
Permutations & No: Proposition 3.18 & No: Theorem 4.3 & No: Theorem 5.5 \\
Words & No: Proposition 3.18 & No: Theorem 4.3 & Yes: Theorem 5.4 \\
\hline
\end{tabular}


Table 4: Summary of results for the M-strong homomorphic image order

\begin{tabular}{lccc}
\hline \hline Class $\mathcal{C}$ & PWO & JPP & DAP \\
\hline Digraphs & No: Theorem 3.1 & No: Theorem 4.4 & Yes: Theorem 5.6 \\
Graphs(reflexive) & No: Theorem 3.3 & No: Theorem 4.5 & Yes: Theorem 5.6 \\
Graphs (irreflexive) & No: Theorem 3.2 & No: Theorem 4.4 & Yes: Theorem 5.6 \\
Trees (reflexive) & No: Remark 3.4 & No: Theorem 4.5 & Yes: Theorem 5.6 \\
Trees (irreflexive) & No: Remark 3.4 & No: Theorem 4.1 & No: Theorem 5.7 \\
Posets (reflexive) & No: Theorem 3.8 & No: Theorem 4.5 & Yes: Theorem 5.6 \\
Posets (irreflexive) & No: Theorem 3.7 & No: Theorem 4.4 & Yes: Theorem 5.6 \\
Tournaments (reflexive) & No: Theorem 3.6 & No: Theorem 4.2 & Yes: Theorem 5.6 \\
Tournaments (irreflexive) & No: Theorem 3.6 & No: Theorem 4.2 & Yes: Theorem 5.6 \\
Equivalence relations & No: Theorem 3.16 & No: Theorem 4.5 & Yes: Theorem 5.6 \\
Linear orders & No: Proposition 3.17 & No: Theorem 4.5 & Yes: Theorem 5.6 \\
Permutations & No: Proposition 3.18 & No: Theorem 4.3 & Yes: Theorem 5.6 \\
Words & No: Proposition 3.18 & No: Theorem 4.3 & Yes: Theorem 5.6 \\
\hline
\end{tabular}

